\section{T T}

Abstract

Recirculating, energy-recovering linacs can be used as driver accelerators for high power FELs. Instabilities which arise from fluctuations of the cavity fields are investigated. Energy changes can cause beam loss on apertures. or, when coupled to $M_{56}$, phase oscillations. Both effects change the beam induced voltage in the cavities and can lead to unstable variations of the accelerating field. Stablity analysis for small perturbations from equilibrium is performed and threshold currents are determined. Design strategies to increase the instability threshold are discussed and the high average power FEL proposed for construction at CEBAF is used as an example.

\section{INTRODLCTION}

Multipass recirculating, energy-recovering accelerators can be cost-effective and energy-efficient driver accelerators to high-average-power, high-efficiency. low-cost FEIs. These accelerators however, are prone to instabilities which arise from fluctuations in energy which in turn cause current loss on apertures, leading to if field changes and further energy changes. Furthermore, energy fiuctuations coupled to compaction factors of non-isochronous arcs can also cause changes in the arrival time of the bunches at the entrance of the linac, leading to further energy changes. These instabilities have been observed experimentally in the energy recovery experiment performed at Los Alamos [1]. Both types of instabilities (longitudinal and beam loss) are analyzed here

We start with the equivalent circuit model for a cavity, and present the equation that describes the interaction between cavity fields and the beam. Next we perform a stability analysis for small perturbations from equilibrium and we derive expressions for threshold currents for both types of instabilities. As a numerical example we use the design parameters of the high average power FEL proposed for construction at CEBAF. We conclude with a discussion on the role of feedback and we outline plans for future work.

\section{EQUIVALENT CIRCUIT MODEL FOR A CAVITY}

An rf cavity powered by an if source (klystron) can be represented by a resonant LCR circuit [2]. The beam in the cavity is represented by a current generator. The interaction of the beam with the cavity fields can be described,

- Work supported by the Virginia Center for Innovative Technology and DOE Contract \# DE-AC05-84ER40150. to a very good approximation, by the following first order differential equation,

$$
\frac{d \bar{V}_{c}}{d t}+\frac{\omega_{0}}{2 Q_{L}}(1-i \tan \Psi) \bar{V}_{c}=\frac{\omega_{0} R_{L}}{2 Q_{L}}\left(\bar{I}_{g}-\bar{I}_{b}\right)
$$

where $\omega_{0}$ is the cavity resonant frequency, $Q_{L}$ is the loaded $Q$ of the cavity and $R_{L}$ is the loaded shunt impedance $R_{L}=(r / Q) Q_{L}$. In arriving at (1) we assume that the cavity voltage, generator and beam current vary as $e^{i \omega t}$, where $\omega$ is the if frequency, and $\bar{V}_{c}, \bar{I}_{g}$ and $\bar{I}_{b}$ are the corresponding complex amplitudes (phasors) in the rotating frame of reference, varying slowly with time. In this equation $I_{b}$ is equal to the average beam current (in the limit of short bunches) and $I_{6}$ denotes the magnitude of $\bar{I}_{b}$. Also $\Psi$ is the tuning angle defined by $\tan \Psi=-2 Q_{L}\left(\omega-\alpha_{0}\right) / \omega_{0}$. In steady-state the generator power is given by

$$
P_{g}=\frac{(I+\beta)}{4 \beta} I_{g}^{2} R_{L} \text {, }
$$

where $\beta$ is the cavity coupling coefficient, and can be calculated from $Q_{L}=Q_{0} /(1+\beta)$.

\section{STABILITY ANALYSIS}

As a concrete example, we take the energy-recovering driver accelerator design of the CEBAF FEL [3]. The generalization is straightforward. We assume that the accelerator consists of a linac with a two-pass recirculation transport. The beam is injected into the linac, accelerated and transported through the first (low energy) arc. It then returns to the linac where it is accelerated for a second time and transported through the second (high energy) arc. A wiggler magnet in the middle of the second arc provides the FEL interaction, as a result of wich the beam loses energy and greatly increases its energy spread. The resulting beam is returned into the linac, decelerated for energy recovery through two passes and then transferred into the dump. Therefore, in this model, there are four beams in the linac cavities at any time (two accelerating and two decelerating).

Furthermore, we limit the stability analysis to the linear regime where perturbations from equilibrium are small. This linear approximation is sufficient for determining the stability boundaries of the system and the growth rates of instabilities. Much of our formalism is similar to the anal$y$ sis of the If stability in the Los Alamos FEL energy recovery experiment [1].

Two effects may trigger an unstable behavior of the system: beam current loss and shift in the arrival time of each pass at the entrance of the linac. The beam current loss may originate from an energy offset which shifts the beam centroid off its central trajectory and leads to beam scraping on apertures. The phase shift may originate from an 
This report hes been reproduced from the best available copy.

Available to DOE and DOE contractors from the Office of Scientific and Technical Information, P.O. Box 62, Oak Ridge, TN-37831; prices available from (615)576-8401, FTS 626-8401.

Available to the public from the National Technical Information Service, U.S. Department of Commerce, 5285 Port Royal Rd., Springfield, VA 22161.

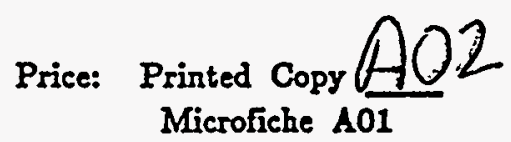




\section{DISCLAIMER}

Portions of this document may be illegible in electronic image products. Images are produced from the best available original document. 
energy offset coupled to the finite compaction factors $\left(M_{56}\right)$ in the arcs.

We assume that the generator current $\tilde{I}_{g}$ is constant and is expressed in the polar form $\bar{I}_{g}=I_{g} e^{i \Psi_{g}}$. We assume that the cavity voltage is perturbed in amplitude and phase. by $\dot{v}(t)$ and $\dot{o}(t)$ respectively, therefore

$$
\bar{V}_{c}=\left[V_{c 0}+\dot{v}(t)\right] e^{i\left[\Psi_{c}+\dot{\phi}(t)\right]} .
$$

We now write the expressions for the beams in each of the four passes. We assume that the two accelerating-pass beams (pass 1 and 2) remain unperturbed and express them in polar form as $\bar{I}_{1}=I_{0} e^{i \Psi_{1}}, \bar{I}_{2}=I_{0} e^{i \Psi_{2}}$, where $\Psi_{1}$ and $\Psi_{2}$ are the beam phases with respect to the phase of the cavity voltage $\Psi_{c}$. However there may be energy errors at the end of each pass given by

$$
\epsilon_{1}(t)=V_{c}(t) \cos \left[\Psi_{c}+\hat{\phi}(t)-\Psi_{1}\right]-V_{c 0} \cos \left(\Psi_{c}-\Psi_{1}\right)
$$

for pass 1 beam, and

$\epsilon_{2}(t)=\epsilon_{1}(t)+V_{c}(t) \cos \left[\Psi_{c}+\dot{\phi}(t)-\Psi_{2}\right]-V_{z 0} \cos \left(\Psi_{c}-\Psi_{2}\right)$

for pass 2 beam. where $V_{c}$ is the magnitude of $\bar{V}_{c}$. The two decelerating beams can be perturbed both in magnitude and phase

$$
\begin{aligned}
& \tilde{I}_{3}=\left[I_{0}+\dot{i}_{3}(t)\right] e^{i\left[\Psi_{3}+\dot{\phi}_{3}(t)\right]} \\
& \tilde{I}_{4}=\left[I_{0}+\dot{i}_{4}(t)\right] e^{i\left[\Psi_{4}+\dot{\varphi}_{4}(t)\right]}
\end{aligned}
$$

where

$$
\begin{gathered}
\dot{i_{3}}(t)=-b_{2} I_{0} \epsilon_{2}\left(t-\tau_{2}\right) \\
\phi_{3}(t)=-h_{2} \epsilon_{2}\left(t-\tau_{2}\right)
\end{gathered}
$$

and $\dot{i}_{4}(t)=\dot{i}_{3}(t), \quad \dot{\phi}_{4}(t)=\phi_{3}(t)$. The coefficients $h_{2}$ and $b_{2}$ are proportional to the compaction factor of the second arc and its momentum aperture respectively, and can be expressed as

$$
h_{2}=\frac{M_{56 \omega}}{E}
$$

where $M_{56}$ is the compaction factor of the arc, $\omega$ is the if frequency, $c$ is the speed of light and $E$ the beam energy around the arc. Similarly, $b_{2}$ can be expressed as

$$
b_{2}=-\frac{\eta_{x}}{L E}
$$

where $\eta_{x}$ is the horizontal dispersion (at the maximum point) of the arc, and $L$ is a loss coefficient which characterizes the amount of beam loss; e.g., if $1 \mathrm{~mm}$ offset gives rise to $10^{-3}$ of beam scraping, then $L=1 \mathrm{~m}$.

In addition we have taken into account the time $\tau_{2}$ it takes the electrons to travel around the second arc and through the linac. To simplify the calculations we have assumed that the first arc is isochronous $\left(h_{1}=0\right)$ and it has "infinite aperture" $\left(b_{1}=0\right)$.
It is assumed that all perturbations imparted to the equilibrium state are small, $\dot{v} \ll V_{c 0}, \dot{\phi} \ll 1, \dot{i}_{3.4} \ll I_{0}$, $\phi_{3.4} \ll 1$. We can therefore linearize eqs (2), (3) to get

$$
\begin{aligned}
\epsilon_{1}(t)= & \dot{v}(t) \cos \left(\Psi_{1}\right)+\hat{\phi} V_{c 0} \sin \left(\Psi_{1}\right) \\
\epsilon_{2}(t)= & \dot{v}(t)\left[\cos \left(\Psi_{1}\right)+\cos \left(\Psi_{2}\right)\right] \\
& +\dot{\phi} V_{c 0}\left[\sin \left(\Psi_{1}\right)+\sin \left(\Psi_{2}\right)\right]
\end{aligned}
$$

where we have set $\Psi_{c}=0$, without loss of generality. From eqs $(4)$ and (5) we write $i_{3}(t)$ and $\phi_{3}(t)$ as

$$
\begin{aligned}
\dot{i}_{3}(t)= & -\hat{v}\left(t-\tau_{2}\right) b_{2} I_{0}\left(\cos \Psi_{1}+\cos \Psi_{2}\right) \\
& -\dot{\phi}\left(t-\tau_{2}\right) b_{2} I_{0} V_{c 0}\left(\sin \Psi_{1}+\sin \Psi_{2}\right) \\
\phi_{3}(t)= & -\dot{v}\left(t-\tau_{2}\right) h_{2}\left(\cos \Psi_{1}+\cos \Psi_{2}\right) \\
& -\dot{\phi}\left(t-\tau_{2}\right) h_{2} V_{c 0}\left(\sin \Psi_{1}+\sin \Psi_{2}\right) .
\end{aligned}
$$

Notice that perturbations on both decelerating beams can be expressed in terms of $\hat{v}$ and $\dot{\phi}$ only through a series of nested relations. This is true for any number of passes and the problem at the end amounts to finding the roots of the determinant of a $2 \times 2$ matrix.

Substituting the above equations into the cavity equation (1), separating real and imaginary parts and performing the linearization, we obtain two linear differential equations in $\dot{v}$ and $\bar{\phi}$. To study the stability of the system we assume that $\bar{v}(t)$ and $\dot{\phi}(t)$ vary with time according to $e^{s t}, \bar{v}(t)=$ $v(s) e^{s t}, \dot{\phi}(t)=\phi(s) e^{s t}$, to obtain two algebraic equations $M A=0$, where $M$ is a $2 \times 2$ matrix and $A$ is the column vector with $v$ and $\phi$ as components. The matrix elements of.$M$ are

$$
\begin{aligned}
& M_{11}=s+\frac{\omega_{0}}{2 Q_{L}}-\frac{\omega_{0} R_{L}}{2 Q_{L}} \cdot A_{1} e^{-s \tau_{2}} \\
& M_{12}=V_{c 0}\left[\frac{\omega_{0}}{2 Q_{L}} \tan \Psi-\frac{\omega_{0} R_{L}}{2 Q_{L}} B_{1} e^{-s \tau_{2}}\right] \\
& M_{21}=-\frac{\omega_{0}}{2 Q_{L}} \tan \Psi+\frac{\omega_{0} R_{L}}{2 Q_{L}} C_{1} e^{-s \tau_{2}} \\
& M_{22}=V_{c 0}\left[s+\frac{\omega_{0}}{2 Q_{L}}+\frac{\omega_{0} R_{L}}{2 Q_{L}} D_{1} e^{-s \tau_{2}}\right]
\end{aligned}
$$

where

$$
\begin{aligned}
A_{1}= & -I_{0} h_{2}\left(\sin \Psi_{3}+\sin \Psi_{4}\right)\left(\cos \Psi_{1}+\cos \Psi_{2}\right) \\
& +I_{0} b_{2}\left(\cos \Psi_{3}+\cos \Psi_{4}\right)\left(\cos \Psi_{1}+\cos \Psi_{2}\right) \\
B_{1}= & -I_{0} h_{2}\left(\sin \Psi_{3}+\sin \Psi_{4}\right)\left(\sin \Psi_{1}+\sin \Psi_{2}\right) \\
& +I_{0} b_{2}\left(\cos \Psi_{3}+\cos \Psi_{4}\right)\left(\sin \Psi_{1}+\sin \Psi_{2}\right) \\
C_{1}= & -I_{0} h_{2}\left(\cos \Psi_{3}+\cos \Psi_{4}\right)\left(\cos \Psi_{1}+\cos \Psi_{2}\right) \\
& -I_{0} b_{2}\left(\sin \Psi_{3}+\sin \Psi_{4}\right)\left(\cos \Psi_{1}+\cos \Psi_{2}\right) \\
D_{1}= & -I_{0} h_{2}\left(\cos \Psi_{3}+\cos \Psi_{4}\right)\left(\sin \Psi_{1}+\sin \Psi_{2}\right) \\
& -I_{0} b_{2}\left(\sin \Psi_{3}+\sin \Psi_{4}\right)\left(\sin \Psi_{1}+\sin \Psi_{2}\right)
\end{aligned}
$$

The determinant of $M$ is then set to zero and the two roots of $s$ are examined. The real parts of the roots will provide the damping or growth rates of perturbations. The imaginary parts of the roots will give the oscillation frequencies relative to the driving if frequency. If both roots have zero 
or negative real parts, the system is stable; otherwise the system is unstable. We found that the 415 ns delay in the high-energy arc does not affect the stability boundaries and growth rates significantly, therefore we set $\tau_{2}=0$, and thus reduced the complexity of the calculation.

Taking this into account, the two roots of $s$ are

$$
s=\left(\frac{\omega_{0}}{2 Q_{L}}\right)\left\{-1+\frac{1}{2} I_{0} R_{L}\left[\left(h_{2} S+b_{2} C\right) \pm \sqrt{\mathrm{Q}}\right]\right\}
$$

where

$$
Q=\sqrt{\left(h_{2} S+b_{2} C\right)^{2}+\frac{4 \tan \Psi}{R_{L} I_{0}}\left(-h_{2} C+b_{2} S\right)-\left(\frac{2 \tan \Psi}{R_{L} I_{0}}\right)^{2}}
$$

and $S$ and $C$ are defined as

$$
\begin{aligned}
& S=\sum_{i=1}^{2}\left[\sin \left(\Psi_{i}-\Psi_{3}\right)+\sin \left(\Psi_{i}-\Psi_{4}\right)\right] \\
& C=\sum_{i=1}^{2}\left[\cos \left(\Psi_{i}-\Psi_{3}\right)+\cos \left(\Psi_{i}-\Psi_{4}\right)\right]
\end{aligned}
$$

From the two roots the one with the positive sign is the limiting one. We can now derive an expression for the threshold current of these instabilities, by setting $s=0$. For the CEBAF FEL parameters, the longitudinal instability threshold current simplifies to

$$
I_{\mathrm{th}}=\frac{c E}{R_{L} \cdot H_{56} \cdot \cos ^{2} \Psi(S-C \tan \Psi)}
$$

where eq.(6) has been used for $h_{2}$. This equation demonstrates possible design strategies for increasing the instability threshold:

1. reducing the shunt impedance of the linac,

2. choosing the off-crest phases such that the $S$ term cancels the $C \tan \Psi$ term,

3. entirely or nearly isochronous arcs, even in the sense of having opposite signs of $M_{56}$ in the two halfs of the arc (before and after the wiggler),

4. lowering the of frequency.

It is worth noting that this expression for the threshold current agrees with the expression derived in reference [4] in its functional dependency on enetgy, frequency, shunt impedance and compaction factor.

The threshold for the beam loss instability is

$$
I_{\mathrm{th}}=\frac{2 E}{R_{L}\left(\eta_{x} / L\right) C},
$$

where eq. (7) has been used for $b_{2}$.

\section{THE HIGH AVERAGE POWER FEL AT CEBAF: AN EXAMPLE}

The driver accelerator for the high average power FEL proposed for construction at $C E B A F$ is a recirculating energy-recovering $200 \mathrm{MeV}, 5 \mathrm{~mA} \mathrm{cw}$ superconducting if (srf) electron accelerator. The accelerator consists of a 10
MeV injector, a $96 \mathrm{MV}$ srf linac with a two-pass recirculation transport which accelerates the beam to $200 \mathrm{MeV}$, followed by energy recovery deceleration through two passes to $\sim 10 \mathrm{MeV}$, and then transport to the dump.

Longitudinal dynamics imposes off-crest operation for the four beams, and that in turn implies that the cavities must be operated off resonance to minimize the required generator power. In the baseline design the phases of the four beams are: $\Psi_{1}=1.8^{\circ}, \Psi_{2}=-13.5^{\circ}, \Psi_{3}=$ $195.3^{\circ}, \Psi_{4}=180^{\circ}$, therefore $S=0.92$ and $C=-3.82$. The optimum tuning angle is $-61.5^{\circ}$. For $M_{56}=-0.47 \mathrm{~m}$ in the high energy arc, and $E=200 \mathrm{MV}, h_{2}$ is equal to $-7.7 \times 10^{-8} \mathrm{rads} / \mathrm{V}$. Assuming $\eta_{x} / L \approx 1, b_{2}=-5 \times 10^{-9}$ $A / V$. For the above value of $h_{2}$, assuming $b_{2}=0$, the threshold for the longitudinal instability is $130 \mu \mathrm{A}$. On the other hand, for $h_{2}=0$ and the assumed value of $b_{2}$, the threshold for the beam loss instability is $1.4 \mathrm{~mA}$. When both instabilities are present, the threshold is dominated by the longitudinal one and is $130 \mu \mathrm{A}$. Clearly at these design parameters the threshold is less than our design goal. therefore active feedback is required to control the instability. Preliminary analysis indicates that the gain of the If feedback loop stabilizes the system.

An alternative lattice design has been developed which has opposite signs of $M_{56}$ for the lattice segments entering and leaving the wiggler (so that the total $M_{56} \sim 0$ ), and the beam phases could be arranged to cancel, therefore onresonance operation would be possible. This alternative design is unconditionally stable against the longitudinal instability because.$H_{56} \approx 0$ over the second arc. For the atternative design $\left(\Psi_{1}=13.5^{\circ}, \Psi_{2}=0^{0}, \Psi_{3}=193.5^{\circ}, \Psi_{4}=\right.$ $180^{\circ}$ ), $S \sim 0$ and $C=-3.9$. However both the baseline and the alternative scenarios have approximately the same beam loss instability threshold.

\section{CONCLUSIONS}

The above analysis is valid only in the open loop case. The presence of feedback will damp the growth of phase oscillations. Since there is no phase shift induced by the instabilities, we expect that maximum gain is needed at the cavity resonance frequency. Gains of $3000(70 \mathrm{~dB})$, which are typical in the CEBAF if control system at these frequencies, will effectively move the threshold well above the $5 \mathrm{~mA}$ design current. Future directions include incorporating feedback in our analysis and addressing gain vs bandwidth questions.

\section{Acknowledgments}

The authors acknowledge the contributions of $S$. Benson and G. Neil to this work.

\section{References}

[1] T.-S. Wang and H. Takeda, PAC Proceedings (1087)

[2] P. B. Wilson, SLAC-PUB-2S84, February 1982.

[3] D. Neuffer et al, these PAC proceedings (1995)

[4] J. J. Bisognano and M. Fripp, Linac Conf Proc (19S8) 